\title{
Duration of triple antithrombotic therapy and outcomes among patients undergoing percutaneous coronary intervention
}

\author{
Rahman Shah $^{1,2}$, Glenda Delgado ${ }^{1}$, Shannon W. Finks ${ }^{3}$ \\ ${ }^{1}$ Section of Cardiology, University of Tennessee, School of Medicine, Memphis, TN, USA; ${ }^{2}$ Veterans Affairs Medical Center, Memphis, TN, USA; \\ ${ }^{3}$ The University of Tennessee, College of Pharmacy, Memphis, TN, USA \\ Correspondence to: Rahman Shah, MD. University of Tennessee, School of Medicine, Section of Cardiovascular Medicine, 1030 Jefferson Avenue, \\ Memphis, TN, USA. Email: Shahcardiology@yahoo.com. \\ Provenance: This is a Guest Editorial commissioned by Deputy Editor-in-Chief Shao-Ping Nie, MD, FESC (Emergency \& Critical Care Center, \\ Beijing Anzhen Hospital, Capital Medical University, Beijing, China). \\ Comment on: Koskinas KC, Räber L, Zanchin T, et al. Duration of Triple Antithrombotic Therapy and Outcomes Among Patients Undergoing \\ Percutaneous Coronary Intervention. JACC Cardiovasc Interv 2016;9:1473-83.
}

Submitted Sep 14, 2016. Accepted for publication Sep 28, 2016.

doi: $10.21037 /$ cdt.2016.11.07

View this article at: http://dx.doi.org/10.21037/cdt.2016.11.07

In the July 2016 issue of $\mathcal{A} A C C$ : Cardiovascular Interventions, Koskinas et al. used observational registry data to compare the safety and efficacy outcomes of triple antithrombotic therapy (TAT) prescribed for one month versus longerduration TAT in patients undergoing percutaneous coronary intervention (PCI) with a clear indication for oral anticoagulation (OAC) therapy (1).

This study retrospectively analyzed data from a cohort of patients with clinical indication for TAT enrolled in the Bern PCI Registry. The registry includes data on 8,772 consecutive patients who underwent PCI between March 2009 and December 2013 in Switzerland. A total of 568 (6\%) patients had clinical indications for OAC of which atrial fibrillation (55\%) was the most common. Among these, $323(56.9 \%)$ were discharged on TAT for one month; the remainder was treated with TAT for longer (median, 3 months; interquartile range, 3 to 6 months). The onemonth treatment was more likely prescribed in those with stable coronary artery disease (CAD), relatively high HASBLED (hypertension, abnormal liver or renal function, stroke or thromboembolism, bleeding history, elderly $>$ age 65 , drug consumption, or alcohol abuse) score, bare metal stent placement, balloon angioplasty, or female gender. A vitamin $\mathrm{K}$ antagonist was the most common OAC (>95\%) utilized. Novel OACs were used more often in the 1-month TAT group than in the others (5.3\% versus $1.9 \%$ ). All patients were discharged on aspirin. Clopidogrel was the predominant $\mathrm{P}_{2} \mathrm{Y}_{12}$ inhibitor; it was prescribed for $99.5 \%$ of those in the 1-month TAT group compared to $96.3 \%$ in the other. The primary endpoint was a composite of cardiac death, MI, stroke, definite stent thrombosis, or TIMI major bleeding. Complete follow-up was available for 533 patients $(93.8 \%)$ at 1 year. At 1 year, the primary composite endpoint occurred in 22 patients $(9.5 \%)$ among the 1-month TAT group and 39 patients (12.9\%) among the others. The difference was not statistically significant after multivariate analysis [adjusted for sex, clinical presentation with ACS, HAS-BLED score, use of any DES, and multiplelesion treatment; adjusted hazard ratio (HR), 1.07; 95\% confidence interval (CI), 0.56-2.06; $\mathrm{P}=0.84]$. These results were consistent in stratified analyses relative to clinical presentation with ACS and PCI with drug-eluting stents (P for interaction $=0.18$ and 0.95 , respectively) as well as in an additional analysis between 1 and 12 months. There were no differences in Bleeding Academic Research Consortium (BARC) bleeding $\geq 3$ (adjusted HR, 0.62; 95\% CI, 0.21-1.80; $\mathrm{P}=0.37$ ) or the secondary composite ischemic endpoint (cardiac death, MI, stroke, or definite stent thrombosis). Furthermore, at 1 month, BARC bleeding $\geq 3$ (adjusted HR, 0.62; 95\% CI, 0.21-1.80; $\mathrm{P}=0.37$ ) and TIMI major bleeding were not significantly different between the two groups.

The authors concluded that after 1 year of clinical follow-up, 1-month TAT therapy (which was used preferentially in patients with higher bleeding risk) was 
associated with similar net clinical outcomes compared with longer TAT therapy.

A major strength of observational studies such as the study by Koskinas et al. is that findings are particularly useful for decision-making in a real-life setting outside the idealized environment of a RCT. However, observational data also have major limitations which influence the application of their findings (2). In observational studies, patient treatment is specifically chosen (i.e., 1 month TAT versus longer duration) rather than randomly assigned, introducing significant bias from confounding factors. Although identifiable differences can be taken into account during data analysis, it is impossible to ascertain whether such adjustments are adequate to overcome biases. Because of this, observational studies must be treated as hypothesisgenerating, and their findings must be confirmed in larger randomized control trials (RCTs). The findings by Koskinas et al. support the hypothesis that patients with high bleeding risk who require OAC after PCI, can reasonably be limited to 1 month TAT without an increased risk in ischemic events. Their data also suggest that in patients with low risk of bleeding, prolonging TAT beyond 1 month does not increase risk of major bleeding. The optimal duration for such therapies is left to future determination.

The cornerstone therapy for patients with coronary artery stents is dual antiplatelet therapy (DAPT) $(3,4)$. Importantly for $5-10 \%$ of these patients, OACs are clearly indicated in addition to DAPT (5). Thus, management of these patients is a major clinical dilemma because the lower risk of ischemic events on TAT is associated with higher risk of major bleeding, and major bleeding itself is a strong predictor of both short- and longer-term mortality post-PCI (5). However, there is no conclusive evidence indicating the best available management strategy; only two small- to moderately-sized RCTs have been conducted in this field to date.

The first RCT was the WOEST (What is the Optimal Antiplatelet and Anticoagulant Therapy in Patients with Oral Anticoagulation and Coronary Stenting) trial in which 573 post-PCI patients were randomized to either OAC plus clopidogrel (double-therapy which omitted traditional aspirin) or OAC plus clopidogrel and aspirin (TAT) (6). The primary study endpoint (any bleeding up to 1 year post-PCI) occurred in $19.4 \%$ patients in the double-therapy group compared to $44.6 \%$ in the TAT group (HR, 0.36; 95\% CI, 0.26-0.50; $\mathrm{P}<0.001)$. In addition, TIMI major bleeding and BARC type 3 bleeding occurred less frequently in the double-therapy group. The combined secondary end point of death, MI, stroke, target-vessel revascularization, or stent thrombosis occurred in $11.1 \%$ of those in the double-therapy group compared to $17.6 \%$ in the TAT group (adjusted HR, 0.56; 95\% CI, 0.35-0.91, P=0.025). Finally, all-cause mortality was significantly $(\mathrm{P}=0.027)$ lower in the double-therapy group $(2.5 \%)$ compared to the TAT group $(6.3 \%)$. However, major criticisms of the WOEST trial were its open-label nature and its small size: only 279 patients received OAC plus clopidogrel, and of these, only about 180 received a DES. Thus, it did not have enough power to detect stent thrombosis risk.

In the second RCT, the ISAR-TRIPLE (Triple Therapy in Patients on Oral Anticoagulation after Drug Eluting Stent Implantation) trial, 604 patients receiving concomitant aspirin and $\mathrm{OAC}$ were randomized to either 6 weeks or 6 months of clopidogrel therapy (7). At 9 months, the primary endpoint (composite of death, MI, definite stent thrombosis, stroke, and TIMI major bleeding) occurred in $9.8 \%$ of those in the 6 -week group and in $8.8 \%$ of those in the 6-month group (HR, 1.14; 95\% CI, 0.68-1.91; $\mathrm{P}=0.63$ ). Similarly, no difference was found in TIMI major bleeding rate $(5.3 \%$ vs. $4.0 \%$; HR, 1.35 ; 95\% CI, $0.64-2.84 ; \mathrm{P}=0.44)$. Although the researchers concluded that 6 weeks of TAT was not superior to 6 months with respect to net clinical outcomes, readers have interpreted that a shorter duration of TAT is acceptable because it does not increase the risk of ischemic events.

Similarly, a recent meta-analysis of 17 observational studies including 14,921 patients showed that TAT therapy, compared to double-therapy, does not decrease risk of recurrent MI, stent thrombosis, and all-cause mortality (8). However, it did increase the risk of major bleeding. Currently, several large-scale trials, such as the PIONEER AF-PCI [A Study Exploring Two Strategies of Rivaroxaban (JNJ39039039; BAY-59-7939) and One of Oral Vitamin K Antagonist in Patients With Atrial Fibrillation Who Undergo Percutaneous Coronary Intervention; NCT01830543] trial, the REDUAL-PCI (Evaluation of Dual Therapy with Dabigatran vs. Triple Therapy with Warfarin in Patients with AF that undergo a PCI with Stenting; NCT02164864) trial, the AUGUSTUS (An Open-label, $2 \times 2$ Factorial, Randomized Controlled, Clinical Trial to Evaluate the Safety of Apixaban vs. Vitamin $\mathrm{K}$ Antagonist and Aspirin vs. Aspirin Placebo in Patients With Atrial Fibrillation and Acute Coronary Syndrome or Percutaneous Coronary Intervention; NCT02415400) trial, and the MUSICA-2 (Anticoagulation in Stent Intervention; NCT01830543) trial are ongoing evaluating double versus 
TAT in contemporary settings that include novel OAC agents as well as warfarin. Further, the optimal antiplatelet therapy (aspirin or $\mathrm{P} 2 \mathrm{Y}_{12}$ ) for double therapy would add value to our current understanding.

In conclusion, current guidelines/evidence suggest that the duration of TAT should be individualized based on the risk of stent thrombosis/ischemic events versus bleeding risk and whether the stent was placed in a setting of stable CAD or ACS $(3,4)$. Patient bleeding risk and stroke risk can be predicted based on HAS BLED and CHA2DS2-VASc, respectively. Factors associated with increased ischemic risk (including stent thrombosis) are advanced age, ACS presentation, multiple prior MIs, extensive CAD, diabetes mellitus, chronic kidney disease, left ventricular ejection fraction less than $40 \%$, first-generation drug-eluting stent, stent undersizing, stent under deployment, small stent diameter, greater stent length, bifurcation stents, and instent restenosis (3). Based on these scores/risk factors, patients with relatively lower bleeding risk and relatively higher stent thrombosis/ischemic event risk should receive longer TAT, while those with the opposite risk profile should receive shorter TAT. For patients with higher risk of both ischemic and bleeding events, individual patient preferences and values should be taken into consideration because individuals may weigh these outcomes differently. Until RCT confirm new information, the preferred $\mathrm{P} 2 \mathrm{Y}_{12}$ inhibitor in TAT is clopidogrel, the target INR is $2-2.5$, the aspirin dose is less than $100 \mathrm{mg}$, and a concurrent protein pump inhibitor should be considered (3).

\section{Acknowledgements}

None.

\section{Footnote}

Conflicts of Interest: The authors have no conflicts of interest to declare.

\section{References}

1. Koskinas KC, Räber L, Zanchin T, et al. Duration of Triple Antithrombotic Therapy and Outcomes Among Patients Undergoing Percutaneous Coronary Intervention. JACC Cardiovasc Interv 2016;9:1473-83.
2. Pocock SJ, Elbourne DR. Randomized trials or observational tribulations? N Engl J Med 2000;342:1907-9.

3. Levine GN, Bates ER, Bittl JA, et al. 2016 ACC/ AHA Guideline Focused Update on Duration of Dual Antiplatelet Therapy in Patients With Coronary Artery Disease: A Report of the American College of Cardiology/ American Heart Association Task Force on Clinical Practice Guidelines. J Am Coll Cardiol 2016;68:1082-115.

4. Lip GY, Windecker S, Huber K, et al. Management of antithrombotic therapy in atrial fibrillation patients presenting with acute coronary syndrome and/or undergoing percutaneous coronary or valve interventions: a joint consensus document of the European Society of Cardiology Working Group on Thrombosis, European Heart Rhythm Association (EHRA), European Association of Percutaneous Cardiovascular Interventions (EAPCI) and European Association of Acute Cardiac Care (ACCA) endorsed by the Heart Rhythm Society (HRS) and AsiaPacific Heart Rhythm Society (APHRS). Eur Heart J 2014:35:3155-79.

5. Schömig A, Sarafoff N, Seyfarth M. Triple antithrombotic management after stent implantation: when and how? Heart 2009;95:1280-5.

6. Dewilde WJ, Oirbans T, Verheugt FW, et al. Use of clopidogrel with or without aspirin in patients taking oral anticoagulant therapy and undergoing percutaneous coronary intervention: an open-label, randomised, controlled trial. Lancet 2013;381:1107-15.

7. Fiedler KA, Maeng M, Mehilli J, et al. Duration of Triple Therapy in Patients Requiring Oral Anticoagulation After Drug-Eluting Stent Implantation: The ISAR-TRIPLE Trial. J Am Coll Cardiol 2015;65:1619-29.

8. Bavishi C, Koulova A, Bangalore S, et al. Evaluation of the efficacy and safety of dual antiplatelet therapy with or without warfarin in patients with a clinical indication for DAPT and chronic anticoagulation: A meta-analysis of observational studies. Catheter Cardiovasc Interv 2016;88:E12-22.

Cite this article as: Shah R, Delgado G, Finks SW. Duration of triple antithrombotic therapy and outcomes among patients undergoing percutaneous coronary intervention. Cardiovasc Diagn Ther 2017;7(Suppl 2):S66-S68. doi: 10.21037/ cdt.2016.11.07 